\title{
Diventare italofono. Un francofono del Senegal stregato dalla lingua di Dante. Walter Zidarič dialoga con Pap Khouma
}

Walter Zidarič e Pap Khouma

\section{(2) OpenEdition}

Journals

Edizione digitale

URL: https://journals.openedition.org/cher/10851

DOI: 10.4000/cher.10851

ISSN: 2803-5992

\section{Editore}

Presses universitaires de Strasbourg

\section{Edizione cartacea}

Data di pubblicazione: 30 juin 2013

Paginazione: 47-53

ISBN: 978-2-35410-054-4

ISSN: 1968-035X

Notizia bibliografica digitale

Walter Zidarič e Pap Khouma, «Diventare italofono. Un francofono del Senegal stregato dalla lingua di Dante. Walter Zidarič dialoga con Pap Khouma», reCHERches [Online], 10 | 2013, online dal 08 février 2022, consultato il 10 février 2022. URL: http://journals.openedition.org/cher/10851 ; DOI: https:// doi.org/10.4000/cher.10851

\section{c) (7) (2) (2)}

Ce(tte) œuvre est mise à disposition selon les termes de la Licence Creative Commons Attribution -

Pas d'Utilisation Commerciale - Partage dans les Mêmes Conditions 4.0 International. 


\title{
Diventare italofono. Un francofono del Senegal stregato dalla lingua di Dante. Walter Zidarič dialoga con Pap Khouma
}

\author{
WALTER ZIDARIČ \\ Université de Nantes \\ Pap Khouma \\ Scrittore
}

Walter Zidarič: Come e quando è approdato alla scrittura? Soltanto al Suo arrivo in Italia oppure era qualcosa che l'attirava da prima?

Pap Khouma: Sono approdato alla scrittura dopo aver partecipato per anni ad iniziative per il riconoscimento dei diritti per gli immigrati. Prima di arrivare in Italia nel 1984 parlavo altre lingue: francese, wolof e inglese, ma non una parola di italiano. A Milano, a quell'epoca, viveva circa una trentina di senegalesi. Nel 1986, con l'aumento progressivo di questa comunità, insieme ad alcuni amici abbiamo creato l'Associazione dei Senegalesi della Lombardia. Organizzavamo riunioni, manifestazioni di piazza, facevamo dichiarazioni alla radio o sui giornali, ma l'Italia continuava ad ignorare la nostra presenza.

Eravamo tutti clandestini, a parte i rifugiati sudamericani, qualche somalo, eritreo e egiziano e pochissimi studenti. Nel 1989 sono stato protagonista di un documentario sulla vita degli stranieri in Italia dal titolo Stranieri tra noi. Un milanese del Senegal racconta, prodotto dallo Studio Equatore. Nello stesso anno il settimanale «Epoca» chiese a me e a un mio amico senegalese, Bathily Dia, di fare un servizio sulla realtà degli immigrati. Insieme a un fotografo abbiamo girato l'Italia da Trieste a Catania, passando da Torino, 
Venezia, Brescia, Milano, Roma, Caserta, Villa Literno. Abbiamo intervistato e fotografato uomini e donne arrivati dall'Africa, dall'Asia e dall'America. L'impatto è stato molto forte perché gli italiani hanno scoperto ciò che fingevano di non vedere: la presenza di stranieri che lavoravano nelle case, nelle fabbriche, nei campi per la raccolta di pomodori... Il mio incontro con Oreste Pivetta è stato decisivo: fu lui a propormi di fare insieme un libro sull'immigrazione e così nacque Io venditori di elefanti, pubblicato nel 1990 da Garzanti ${ }^{1}$. Attualmente il libro è rieditato dalla Baldini \& Castoldi Dalai e quest'anno è stato tradotto in inglese per l'Indiana University Press con il titolo I was an elephant salesman².

\section{W.Z.: Che cosa rappresenta per Lei, intimamente, la scrittura?}

P.K.: Il primo libro, Io venditore di elefanti, ha avuto per me un ruolo quasi taumaturgico. Avevo vissuto circa tre anni di clandestinità ed è stata un'esperienza angosciosa che ti segna per il resto della vita. Essere clandestino in Italia significava, all'epoca, non avere nessun diritto ed essere in balìa dei capricci e degli abusi della gente comune, bambini, adulti, uomini in divisa: scherni quotidiani, arresti con accuse pretestuose, condanne, fogli di via, cioè mandati di espulsione dal territorio nazionale. Sono stato spesso arrestato e messo in cella o mandato direttamente in carcere senza aver commesso un delitto. Ho ricevuto più volte l'ingiunzione di lasciare l'Italia, ma non la potevo rispettare perché non avevo i soldi per comprare il biglietto d'aereo. Il clandestino non poteva cercare un lavoro né pretendere di affittare una casa. Era quasi impossibile ritornare nel paese di origine anche volontariamente, per mancanza di soldi. Io venditore di elefanti, che narra questi fatti, è stato definito dalla critica come un importante sasso nello stagno della storia dell'immigrazione in Italia. È un libro raccontato e scritto in maniera semplice, ma è riuscito, attraverso l'esperienza di alcuni, a dipingere in profondità la vita di tanti giovani.

Attualmente l'Italia è diventata un paese multiculturale come Francia, Belgio, Inghilterra ma la nostra classe politica tende a sottovalutare o addirittura a negare quest'evidenza. In generale i conflitti sociali e culturali

1 P. Khouma, Io venditore di elefanti. Una vita per forza tra Dakar, Parigi e Milano, a cura di O. Pivetta, Milano, Garzanti, 1990 (1a ed.); Milano, Baldini \& Castoldi Dalai, 2005 (2a ed.), 2010 ( $3 \mathrm{a}$ ed.).

2 Id., I was en elephant salesman. Adventures Between Dakar, Paris and Milan, edited by Oreste Pivetta, translated by Rebecca Hopkins, with an introduction by Graziella Parati, Indiana, Indiana University Press, 2010. 
che ne scaturiscono sono affrontati in maniera strumentale. Un intellettuale, soprattutto se è uno scrittore immigrato, ha il dovere di affrontare caparbiamente, ma senza perdere la lucidità, i conflitti socioculturali e di proporre delle soluzioni. Scrivo per mantenere il dialogo tra autoctoni e immigrati, per cercare di dar voce ai tanti stranieri di diverse origini che non ce l'hanno, per sviscerare i problemi sociali e prospettare delle soluzioni. Partecipo a tanti convegni in Italia o all'estero e insieme alla scrittura di libri, poesie o articoli, agli interventi alla radio e alla televisione, provo a dare un'immagine "normale" e non stereotipata degli immigrati in generale.

W.Z.: Lei è stato uno fra i primi, all'inizio degli anni 1990, ad aprire la strada di quella che è stata definita in un primo tempo Letteratura della migrazione. Che sguardo porta adesso su quel periodo e sul suo primo libro, Io, venditore di elefanti, scritto in collaborazione con Oreste Pivetta?

P. K.: Negli anni Ottanta, ricercatori, sociologi e scrittori italiani avevano iniziato a raccontare e a pubblicare la vita degli immigrati. Illustri mâttres à penser come Giorgio Bocca o Indro Montanelli hanno scritto all'epoca libri $\mathrm{o}$ articoli generalizzanti, pieni di preconcetti sulla realtà degli immigrati. Gli articoli o le notizie alla radio o alla televisione erano spesso approssimativi. Io come altri (Salah Methani, Saidou Moussa Ba, Nacera Sohra, Mohamed Bouchane, Kossi Komla Ebri, Tahar Lamri, ecc.) avevo urgenza di raccontare in prima persona l'immigrazione agli italiani. Insieme agli altri stranieri operai, venditori, impiegati, eravamo nostro malgrado protagonisti di cambiamenti sociali, culturali e politici di cui era difficile misurare la portata. Personalmente non possedevo ancora tutti gli strumenti della lingua italiana per poter esprimermi autonomamente. Ripeto, le mie lingue erano il francese e il wolof. L'apporto del giornalista Oreste Pivetta, per quanto mi riguarda, è stato fondamentale. Io venditori di elefanti è classificato tra $\mathrm{i}$ libri scritti a quattro mani ed è stato subito adottato come libro di testo nelle scuole italiane.

W.Z.: Tra il suo primo libro e l'ultimo, Nonno Dio e gli spiriti danzanti, sono trascorsi quindici anni. Come mai tanto tempo per tornare alla scrittura?

P. K.: Dopo il 1990 ho iniziato a collaborare con diversi quotidiani e riviste, "L’Unità", "Epoca" e soprattutto "Linus", una rivista satirica sulla quale avevo una rubrica mensile fissa. Sembrava passata la "moda" dei libri scritti a quattro mani, la grande editoria si era praticamente disinteressata della 
letteratura della "migrazione". Nel frattempo erano nate delle piccole case editrici specializzate in questo genere letterario che hanno pubblicato dei testi interessanti e in ogni caso mantenuta viva la fiamma. Volevo ripubblicare in una casa editrice tradizionale, rompere la loro indifferenza nei confronti degli scrittori migranti e dimostrare che, anche se non siamo nati in Italia, siamo lo stesso capaci di scrivere come qualsiasi autore. Finalmente, nel 2005, ho pubblicato un nuovo romanzo: Nonno Dio e gli spiriti danzanti ${ }^{3}$. Ora la grande editoria sembra aver superato i suoi tentennamenti e tanti scrittori migranti vengono di nuovo pubblicati.

W.Z.: Se nel suo primo libro il tema principale era il difficile approdo in Italia di un immigrato senegalese, in Nonno Dio e gli spiriti danzanti si assiste invece al difficile ritorno nel paese natale, il Senegal, del protagonista. Perché la scelta di raccontare questo tipo di storia?

P. K.: Il tema del romanzo Nonno Dio e gli spiriti danzanti non è soltanto quello del ritorno nel paese di origine. È un romanzo in cui fioriscono personaggi non migranti, uomini, donne, spiriti buoni e malvagi che si battono per vivere nella propria terra. $\mathrm{E}$ ambientato in un paese non ben delimitato, il Sahel, che nel romanzo viene ribattezzato Sahael. Non è autobiografico, contrariamente a Io venditore di elefanti. Certo, ci sono le storie di due personaggi che da Milano ritornano a Taagh, capitale del Sahael, e si ritrovano in una spirale di eventi che non controllano. Le vere protagoniste del romanzo sono le donne del Sahael. Sono gli unici personaggi veramente positivi nell'intera narrazione. Nonno Dio e gli spiriti danzanti è un omaggio alla donna africana. In generale le donne africane non sono corrotte come la maggior parte dei maschi africani, non causano le guerre, non sfruttano i bambini e sono le antiche e moderne custodi della famiglia. Purtroppo sono escluse dalla gestione del potere, ma quando ricoprono il ruolo di responsabilità pubbliche vengono unanimemente apprezzate: Ellen Johnson-Sirleaf, Presidente della Liberia; Ngozi OkongoIweala, nigeriana, direttrice generale della Banca Mondiale; Fatou Bensouda, gambiana, magistrata alla Corte penale internazionale; Wangari Maathai, keniota, Premio Nobel per la pace... L'Africa è stata logorata da secoli di schiavitù e colonizzazione e dopo, dittature, carestie, guerre civili, esodi forzati e massici hanno contribuito ad affondarla. A mio parere - ma è anche quello di illustri personaggi, come del nigeriano Wole Sohinka, Premio

3 P. Khouma, Nonno Dio e gli spiriti danzanti, Milano, Baldini \& Castoldi Dalai, 2005. 
Nobel per la letteratura, del grande scrittore ivoriano Ahmadou Kourouma, del leggendario attore burkinabé Sotigui Kouyaté, recentemente scomparso, del regista senegalese Sembene Ousmane - se l'Africa è ancora in piedi, se, malgrado tutto, i suoi figli continuano a sperare, il merito è soprattutto della Donna africana, della sua grande intelligenza, del suo rifiuto della violenza, purtroppo molto diffusa, come ho voluto rappresentare nel libro e come è nella realtà quotidiana di quasi tutti i paesi africani. Questo è anche il senso di Nonno Dio e gli spiriti danzanti.

W.Z.: Il protagonista, Og Dem, si trova nella situazione di essere ormai fra due culture, due paesi, due famiglie, due identità. Non sembra riconoscersi più in quella delle origini, così distante da quella italiana, ma non sembra neppure essere troppo a suo agio con quest'ultima.

P. K.: Og Dem è soltanto il filo conduttore di Nonno Dio e gli spiriti danzanti che, suo malgrado, lega la sua cultura d'origine e il paese dove è nato a quella italiana, dove ha vissuto per tanti anni. Come tutti gli immigrati, Og Dem e il suo amico e cognato Birago hanno volenti o nolenti una doppia o una tripla identità che non sempre rappresentano un vantaggio. A Milano vivevano la nostalgia e i disagi procurati dallo status del migrante, anche se Og ha un buon lavoro in ospedale. Hanno la sfortuna di essere capitati in Sahael in un periodo di turbolenza e di subirne le conseguenze. Nella vita reale all'immigrato può facilmente capitare di vivere dei conflitti interni. $\grave{E}$ facile sentirsi a disagio nel paese di approdo senza riconoscersi più in quello di origine. Questo capita ai personaggi Og Dem e Birago.

W.Z.: Se nel suo primo libro Lei si era avvalso dell'aiuto di un giornalista italiano, in questo, invece, che forse potrebbe essere considerato come un romanzo di formazione, lei fa uso di plurilinguismo, forse anche di una certa polifonia. Difatti è stato necessario, mi sembra, introdurre varie note esplicative perché il lettore potesse cogliere a fondo il senso della storia; tuttavia rimangono espressioni in francese $e$ in inglese che non sono state tradotte. Pensa che ciò non costituisca una difficoltà per il lettore italiano/ italofono? Qual è il tipo di pubblico a cui si rivolge in particolare?

P. K.: Nonno Dio e gli spiriti danzanti è stato scritto senza ausilio linguistico esterno. Va detto che chi scrive un romanzo, o altro, potrebbe aver bisogno di persone, di amici o della propria compagna per riletture, confronti, suggerimenti. Poi sarà l'autore a decidere se prendere in considerazione gli 
spunti nati dalla discussione. Alcuni hanno definito Nonno Dio e gli spiriti danzanti un romanzo di formazione. Ciò che è fondamentale - faccio anche il mestiere di libraio - è che il lettore di un libro, di qualsiasi genere esso sia, non dovrebbe annoiarsi. Il registro polifonico è dovuto al fatto che ho una pluri-identità. Sono nato senegalese, di madrelingua wolof e di religione musulmana; sin da piccolo sono stato educato nella scuola francese laica; vivo nell'Italia cattolica da più di un quarto di secolo e scrivo racconti e articoli in lingua italiana. Un senegalese, un filippino, un rumeno, un brasiliano, in Italia sono, per forza, plurilingui. Spesso, nello stesso discorso, passano dalla lingua madre a quella di approdo per concludere con la "lingua padre", cioè quella del colonizzatore, almeno nel caso di tanti africani. Ho cercato di rendere questo "plurilinguismo" in Nonno Dio e gli spiriti danzanti, senza troppo esagerare per evitare di disorientare il lettore italiano. Inoltre ho elaborato mentalmente alcuni capitoli del romanzo nelle lingue wolof e francese e li ho successivamente "tradotti", trascritti in lingua italiana mantenendo più o meno la struttura sintattica, le forme retoriche del wolof $o$ del francese. Questo succede soprattutto quando gli episodi sono ambientati in Africa, quando parlano le madri del Sahael, quando entra in scena il coro delle mauvaises langues, quando si inalbera la sacerdotessa del n'depp. I secondini del carcere di Taagh usano un linguaggio "professionale"... in francese, anche gli autisti dei cars-rapides, i trasporti pubblici, possiedono un vivace codice linguistico e nello stesso discorso mischiano wolof, peul, serere e francese, lingue che presentano delle morfologie diverse. Come autore ho tentato di riprodurre in italiano queste particolarità linguistiche.

In ogni caso scrivo per rivolgermi a un pubblico italiano e straniero. Purtroppo, troppi immigrati in Italia hanno problemi quotidiani e urgenti da affrontare e probabilmente il libro rimane ancora un lusso per loro. La maggior parte dei miei lettori è italiana.

W.Z.: Lei è una delle personalità più attive all'interno di questa nuova letteratura che io definirei semplicemente italofona, quale sguardo porta su di essa? Quali prospettive vede per il futuro?

P. K.: Io venditore di elefanti si conclude con la frase: «Nascono bambini». Questi bambini, figli della migrazione, sono ormai cresciuti e sono di madrelingua italiana, perché sono stati educati nella scuola italiana e alcuni di loro pubblicano libri o scrivono articoli sui giornali (come Randa Ghazi o Igiaba Sciego, per esempio). Esistono delle riviste di letteratura italofona soprattutto telematiche, come El-Ghibli, di cui sono il direttore da ormai 
otto anni, Sagarana, diretta da Julio Monteiro, Kumá, diretta da Armando Gnisci. L’Università di Roma "La Sapienza" ha recensito circa cinquecento scrittori italofoni e più della metà è rappresentata da donne. Sono dati confortanti. I testi degli scrittori italofoni vengono tradotti in altre lingue.

\section{W.Z.: Com'è nato il Suo ultimo libro?}

P. K.: Il libro si intitola Noi italiani neri, con il sottotitolo Storie di ordinario razzismo ${ }^{4}$. Non è autobiografico ed è tra il saggio e il romanzo. Tratta il tema della cittadinanza dei figli della migrazione, della discriminazione diffusa nei loro confronti. Pone delle domande sulla violenza legata al razzismo contro i calciatori neri negli stadi italiani, francesi, inglesi. Ho fatto delle ricerche a Milano, Parigi, Barcellona, città dove ho raccolto le testimonianze di giovani nati in Europa ma che hanno la pelle nera o un nome o un cognome considerati non europei. La loro quotidianità è fatta di amore e di amarezza verso il loro paese di nascita, spesso di porte sbattute in faccia; di difficili ricerche di radici lontane, quelle dei genitori o dei nonni; di smarrimento d'identità... Ci sono dei capitoli che rievocano la partecipazione di soldati africani alla prima e alla seconda guerra mondiale. Dopo la fine dei conflitti furono discriminati e dimenticati dalla storiografia europea. C’è un legame tra quei soldati e i nuovi europei con la pelle nera. I soldati africani, venuti a sacrificare la propria vita per la libertà dell'Europa, erano i nonni, gli zii, i padri della maggior parte degli attuali immigrati in Europa. Mio padre e mio zio facevano parte di quei giovani africani venuti a combattere e a morire quando il nazifascismo aveva invaso l'Europa. Ci furono circa 60.000 caduti originari dell'Africa sub-sahariana. Il loro ruolo fondamentale nella vittoria degli alleati fu subito trascurato.

4 Id., Noi italiani neri. Storie di ordinario razzismo, Milano, Baldini \& Castoldi Dalai, 2010. 